\title{
BRIX1 Promotes Ribosome Synthesis and Enhances Glycolysis by Selected Translation of GLUT1 in Colorectal Cancer
}

\section{Chunhui Jiang}

Shanghai Jiao Tong University School of Medicine Affiliated Renji Hospital

Chunjie Xu

Shanghai Jiao Tong University School of Medicine Affiliated Renji Hospital

Siyuan Wen

Shanghai Jiao Tong University School of Medicine Affiliated Renji Hospital

Hanbing Xue

Shanghai Jiao Tong University School of Medicine Affiliated Renji Hospital

Qing Xu ( $\nabla$ renjixuqing@163.com)

Shanghai Jiao Tong University School of Medicine Affiliated Renji Hospital

\section{Research}

Keywords: Colorectal cancer, Ribosome biogenesis protein BRX1 homolog, Ribosome synthesis, GLUT1, Selecting translation

Posted Date: August 3rd, 2021

DOI: https://doi.org/10.21203/rs.3.rs-713733/v1

License: (1) This work is licensed under a Creative Commons Attribution 4.0 International License. Read Full License 


\section{Abstract}

Background: Ribosome biogenesis protein BRX1 homolog (BRIX1) is a vital member for synthesizing $60 \mathrm{~S}$ subunit of ribosome. However, the role and mechanism of BRIX1 in colorectal cancer (CRC) are still unclear.

Methods: KEGG pathway analysis and Gene Ontology (GO) analysis were used for bioinformatics analysis. Ribosome RNAs (rRNAs) levels were detected in CRC tissues and cells. Nascent RNA synthesis were detected by cellular immunofluorescence. Correlation was analysis between PET-CT value and BRIX1 expression. Extracellular acidification rate (ECAR) and oxygen consumption rate (OCR) were conducted by seahorse analyses. Polysome fractions were collected for BRIX1 mRNA used in translation. Orthotopic model and CCK-8 assay were used for BRIX1 function in CRC.

Results: We found that BRIX1 is a core gene of ribosome-related pathway changes in CRC. GO analysis showed BRIX1 primarily enriched RIBOSOME ASSEMBLY and RIBOSOME BIOGENESIS pathways. In fresh CRC tissue, rRNAs levels (5S, 5.8S, 18S and 28S) were obvious higher in BRIX1-high group than BRIX1Low group. Similarly, BRIX1 knockdown and overexpression significantly dereased and increased rRNAs levels (5S, 5.8S, 18S and 28S) in CRC cells, respectively. In addition, BRIX1 knockdown inhibited nascent RNA synthesis of CRC cells. In clinical data analysis, we found that BRIX1 expression is related to the glucose uptake of PET-CT. Consistently, BRIX1 knockdown and overexpression significantly dereased and increased ECAR valve, glucose uptake and lactic acid production in CRC cells. Subsequently, we found BRIX1 knockdown and overexpression significantly dereased and increased the protein expression of GLUT1, but not affect its mRNA expression. Interestingly, we found BRIX1 knockdown and overexpression obviously decreased and increased mRNA level of GLUT1 in polysome fractions. Blocking glycolysis by si-GLUT1 or galactose reversed the promoting role of BRIX1 in glycolysis and cell proliferation of CRC cells.

Conclusion: In this study, BRIX1 plays a tumor-promoting role in CRC via regulating glycolysis by selecting GLUT1 translation, indicating a correlation between ribosome activation and metabolic reprogramming in CRC.

\section{Background}

Colorectal cancer (CRC) is one of the malignant tumors of the digestive tract with the highest incidence and fatality rate(1). Globally, this caused a lot of new cases and fatal cases each year. Since the onset of CRC is hidden and there are no specific symptoms in the early stage, more than $50 \%$ of CRC patients are in advanced stages (stage III and IV) when they are diagnosed, and the overall prognosis of advanced CRC patients is obviously worse than CRC patients with earlier stages $(2,3)$. Therefore, studying and exploring the molecular mechanism of CRC development is a long-term unchanging theme. These efforts are conducive to the development of new methods for the diagnosis and treatment of CRC. 
Ribosomes, as the general factory of protein synthesis, are the basic elements for maintaining the normal life activities of cells. In normal human cells, ribosome synthesis is a dynamic and orderly process, which is precisely regulated to ensure the normal operation of ribosome functions and meet the needs of different life activities(4), while in malignant tumor cells, ribosome synthesis is significantly activated to meet the needs of a large number of structural and functional proteins required for rapid cell division and proliferation $(5,6)$. Pathologically, the increase in nucleolar and nucleolar organization caused by abnormal activation of ribosome synthesis is a characteristic phenotype of malignant tumor cells, indicating the degree of malignancy and prognosis of the tumor(7), and several studies also found ribose in malignant tumor cells. The number of bodies is significantly higher than that of normal cells. Ribosome biogenesis protein BRX1 homolog (BRIX1) is a vital member for synthesizing 60S subunit of ribosome, which located in the nucleus. In the research field of malignant tumor, studies only have reported that the expression of BRIX1 is elevated in non-small cell lung cancer(8), and the role and mechanism of BRIX1 in CRC are still unclear.

Metabolic reprogramming is also one of the characteristic signs of tumor cells $(9-11)$. Changes in the tumor microenvironment determine the direction of tumor cell metabolic reprogramming(12). Abnormal glucose metabolism is a common phenomenon in the metabolic reprogramming of colorectal cancer cells(13). In 1920, Warburg found that the glycolytic ability of liver cancer cells was significantly higher than that of normal liver cells. Interestingly, even in the case of sufficient oxygen, the glycolysis of liver cancer cells is also highly active, called the Warburg effect. Subsequent studies have found that abnormal activation of glycolysis is essential for the proliferation and growth of tumor cells $(14,15)$. However, the related mechanisms regulating glycolysis need to be further explored.

In this study, we found that BRIX1 regulated ribosome activation and determined a high glycolytic state by selected translation for GLUT1, which promoted CRC process. These results indicated a correlation between ribosome activation and metabolic reprogramming in CRC.

\section{Materials And Methods}

\section{Patients and samples}

A total of 392 paraffin sections of CRC tissues and adjacent paired non-cancerous tissues were collected to design a tissue array chip from the Department of Gastrointestinal Surgery, Renji Hospital, School of Medicine, Shanghai Jiao Tong University. All patients with CRC underwent surgery at the Department of Gastrointestinal Surgery, Renji Hospital, School of Medicine, Shanghai Jiao Tong University between January 2014 and January 2016. The study was approved by the Research Ethics Committee of Renji Hospital and carried out in accordance with ethical standards as formulated in the Helsinki Declaration. Informed consents were provided by all patients.

\section{Cell culture}


All cell lines used in this study were obtained from the Cell Bank of the Chinese Academy of Sciences (Shanghai, China). All cell lines were cultured in Dulbecco's modified Eagle's medium (DMEM) supplemented with $10 \%$ fetal bovine serum (FBS) and 1\% penicillin and streptomycin.

\section{Small-interfering RNA (siRNA) transfection}

The siRNAs for GLUT1 were purchased from GenePharma (Shanghai GenePharma Co., Ltd., Shanghai, China). Sequences are shown in Table S1 and experimental method was performed as previously described(16).

\section{Lentivirus transfection}

Full-length human BRIX1 cDNA was transfected into $\mathrm{CRC}$ cell lines using a lentivirus to generate Lentivirus-BRIX1 (BRIX1 overexpression). Lentivirus-NC was used as the negative control (Vector). In addition, one short-hairpin RNA (shRNA) sequence against BRIX1 was transfected into CRC cell lines to generate shRNA-BRIX1, while shNC was used as the negative control. Sequences are shown in Table S1.

\section{RNA isolation and RT-qPCR}

Trizol was used to extract RNA and total RNA was reverse transcribed to cDNA by PrimeScriptTM (TAKARA). We used 18S RNA as an internal control. The sequences of the primers used are shown in Table S1. The relative expression of the target gene was calculated by the $-\triangle \triangle \mathrm{Ct}$ method.

\section{Western blot analysis}

Total protein was extracted using radioimmunoprecipitation assay (RIPA) buffer supplemented with $1 \%$ protease inhibitors and phosphatase inhibitors. Protein concentration was measured with the bicinchoninic acid (BCA) assay. Western blot analysis was performed as previously described(16). BRIX1 (sc-373680, Santa Cruz), GLUT1 (ab115730, Abcam), ß-catenin (ab32572, Abcam), Ki67 (Proteintech Group, Inc.) primary antibodies were used. Horseradish peroxidase (HRP)-conjugated Affinipure Goat AntiRabbit IgG $(\mathrm{H}+\mathrm{L})$ and HRP-conjugated Affinipure Goat Anti-Mouse IgG $(\mathrm{H}+\mathrm{L})$ were obtained from Proteintech Group, Inc (Jackson).

\section{Immunohistochemistry (IHC)}

All tissues were paraffin-embedded and cut into 4-m thick sections. All sections were dewaxed with xylene and hydrated with alcohol. Sodium citrate was used for antigen retrieval and $0.3 \%$ hydrogen peroxide (H2O2) was used to block endogenous peroxidase. After blocking non-specific sites with bovine serum albumin (BSA), all the sections were incubated with an appropriate primary antibody and secondary antibody. We used 3,3-diaminobenzidine (DAB) kit for visualization and hematoxylin was used to stain nuclei. All the sections were dehydrated with alcohol and sealed with neutral resin. IHC staining score was calculated based on pixel intensity; staining was scored as follows as per the staining intensity: no staining, 1; weak staining, 2; moderate staining, 3; and strong staining, 4. 


\section{Seahorse analyses}

The Seahorse XF96 Flux Analyzer (Seahorse Bioscience, Agilent) was used to carry out the extracellular acidification rate (ECAR) and oxygen consumption rate (OCR) in the CRC cell lines. Briefly, all CRC cells used in this paper, including HCT-116, SW480 and HT29 cells, were seeded into an XF96-well plate. The media were replaced with assay media $1 \mathrm{hr}$ before the assay. For ECAR assay (Seahorse Cat.\#103020100), $10 \mathrm{mM}$ glucose, $1 \mu \mathrm{M}$ oligomycin, and $50 \mathrm{mM}$ 2-deoxyglucose (2-DG) were added to the wells. For the OCR test (Seahorse Cat.\#103015-100), $1 \mu \mathrm{M}$ oligomycin, $1 \mu \mathrm{M}$ FCCP, $0.5 \mu \mathrm{M}$ rotenone, and $0.5 \mu \mathrm{M}$ actinycin A were added to the wells at a special time point. Both measurements were normalized by total protein quantitation. The above experiments were performed in triplicate and repeated twice.

\section{Glucose and lactate measurement}

The Amplex® Red Glucose/Glucose Oxidase Assay Kit (Invitrogen, Cat.\#A22189) was used to measure the glucose uptake. Glucose consumption was calculated by the net content of the original glucose concentration deduced the measured glucose concentration in the medium. The Lactate Assay Kit (BioVision, Cat.\#ABIN411683) was used to measure lactate production. Total proteins were used for the normalization of the results obtained above. These experiments were performed in triplicate and repeated twice.

\section{CCK8 assay}

$1 \times 10^{3}$ cells were seeded into 96-well plates. Adding detection reagent $(10 \mu \mathrm{l} \mathrm{CCK8}$ reagent+ $90 \mu \mathrm{l}$ DMEM) to each plate and incubating at $37^{\circ} \mathrm{Cfor} 1 \mathrm{~h}$. Measure the absorbance at $450 \mathrm{~nm}$ with a microplate reader. Each experiment was carried out independently in triplicate.

\section{Polysome fractionation and RNA isolation}

cellular extracts were centrifuged at $10,000 \mathrm{rpm}$ for $5 \mathrm{~min}$ at $4^{\circ} \mathrm{C}$ and the supernatant was carefully isolated and loaded onto $10-50 \%$ sucrose gradients containing $0.1 \mathrm{mg} / \mathrm{ml}$ heparin and $2 \mathrm{mM}$ DTT and centrifuged at $37,000 \mathrm{rpm}$ for $2.5 \mathrm{~h}$ at $4^{\circ} \mathrm{C}$ (SW40 rotor). The sucrose gradient was subsequently fractionated with a gradient fractionation system (ISCO) connected to a UV detector to monitoring absorbance at $252 \mathrm{~nm}$. RNA was isolated from polysomal fractions using the PureLink RNA Mini Kit (Invitrogen).

\section{Animal model}

For the generation of an orthotopic model of CRC, all nude mice were anesthetized with $0.5 \%$ pentobarbital. After opening the abdominal cavity, $1 \times 10^{6} \mathrm{SW} 480^{\text {luc }}$ cells/null mouse were injected into the ileocecum. Tumor growth was monitored by animal CT imaging. After 4 weeks, the mice were sacrificed and the tumor tissues were excised and weighed. All tissues were fixed with $4 \%$ paraformaldehyde. All animal experiments were approved by the Research Ethics Committee of Renji Hospital and adhered to the local or national requirements for the care and use of laboratory animals. 


\section{Statistical analysis}

Measurement data are presented as the mean \pm standard deviation (SD). SPSS 20.0 (Chicago, IL, USA) and GraphPad Prism 7 software were used to conduct the statistical analyses. The correlation of BRIX1 expression with categorical clinical variables in patients with CRC was evaluated using chi-square analysis or Student's t-test. Measurement data, such as age and tumor size, were evaluated using Student's t-test, while categorical variables and ranked data, such as gender, T stage, lymph node invasion, and distant metastasis, were analyzed using the chi-square test. Spearman's rank correlation was used for the analysis of two-way ordered categorical data. Survival curves were generated using the Kaplan-Meier method and analyzed by the log-rank test. Statistical significance was accepted at $\mathrm{p}<$ 0.05 .

\section{Results}

\section{Ribosome-related pathways are primary enriched in CRC}

Due to a KEGG pathway analysis, we found ribosome biosynthesis in eukaryotes and ribosome pathways were primary enriched in CRC samples in GEO datasets (GSE21510 and GSE18105) (Figure 1A). 55 genes were significant expressed in ribosome biosynthesis in eukaryotes and ribosome pathways. Then, we put these 55 genes to protein protein interaction network (PPI) and tried to select key genes (degree $>30$ ) of aberrant activation of ribosome biosynthesis in CRC. In this analysis, we found 8 key genes, including CMSS1, BRIX1, RPS7, RPS20, RPS25, RPL5, RPL11, WDR36, WDR43 and UTP14A, which maybe show primary significance in aberrant activation of ribosome biosynthesis (Figure 1B). Due to further mRNA expression analysis from TCGA dataset, all these 8 genes were obviously upregulated in CRC samples as compared with normal samples (Figure 1C, 1D). These results indicating abnormal activation of ribosome was a significant feature in CRC. We further detected rRNAs in paired CRC tissues and adjacent normal tissues and results showed rRNA expression were obviously increased in CRC tissues compared with adjacent normal tissues (Figure 1E). Similarly, in cell lines, rRNA expression was significantly higher in CRC cell lines than in normal colorectal cell line (Figure S1A-S1D).

\section{BRIX1 welly predicts ribosome activation in CRC}

Due to $G O$ analysis from TCGA dataset (Figure 2A), we found BRIX1 primarily enriched GO_RIBOSOME_ASSEMBLY and GO_RIBOSOME_BIOGENESIS (Figure 2B), indicating the forecast accuracy in abnormal ribosome activation in CRC. Due to detect ribosomes quantity, we found ribosomes quantity were higher in BRIX1-high expressed CRC tissues than BRIX1-low expressed CRC tissue (Figure 2C). Next, we knockdown BRIX1 in HCT-116 and SW480 cell lines (Figure 2D). Similarly, BRIX1 knockdown showed a significant decrease in rRNA levels in shBRIX1 CRC cell (Figure 2E). Moreover, BRIX1 knockdown showed .a significant decrease in nascent RNA synthesis of CRC cells (Figure 2F). These results showed that BRIX1 play a vital role in regulating ribosome activation in CRC.

\section{BRIX1 determines a high glycolytic state in CRC cells}


In the analysis of PET-CT, we found that the glucose uptake of CRC patients with high BRIX1 expression was significantly higher than that of patients with low BRIX1 expression (Figure 3A), which indicates that BRIX1 is related to glucose metabolism. In vitro cell experiments, knocking down BRIX1 significantly decreased the ECAR level in CRC cells (Figure 3B), but had no significant effect on the OCR level (Figure 3C). In addition, knocking down BRIX1 significantly reduces the glucose uptake capacity and reduces the production of lactic acid in CRC cells (Figure 3D, 3E). However, BRIX1 overexpression significantly increased the ECAR level in CRC cells, but aslo had no significant effect on the OCR level (Figure 3F). Moreover, BRIX1 overexpression significantly promotes the glucose uptake capacity and increased the production of lactic acid in CRC cells (Figure 3G).

\section{BRIX1 regulates glycolysis in CRC cells via selected translation for GLUT1}

The above experiments indicate that BRIX1 might control the glycolytic process by regulating glucose uptake in CRC. GLUT1 is a key transporter for glucose uptake. There was no correlation between BRIX1 and GLUT1 expression from TCGA dataset (Figure 4A). In vitro experiment, BRIX1 knockdown does not affect the mRNA expression GLUT1 (Figure 4B). However, BRIX1 knockdown significantly down-regulated GLUT1 protein levels (Figure 4D). Similarly, BRIX1 overexpression also only regulated protein expression of BRIX1, but not mRNA expression (Figure $4 \mathrm{C}, 4 \mathrm{E}$ ). The relative translation rate of an mRNA can be inferred from the number of ribosomes (polysomes) it recruits and can be quantitatively analyzed by employing sucrose gradients to purify mRNAs associated with translationally active ribosomes (Figure 4F). Due to this approach, we collected polysome fractions and the results showed BRIX1 knockdown obviously decreased mRNA level of GLUT1 in polysome fractions (Figure 4G). Contrary, BRIX1 overexpression remarkably increased mRNA level of GLUT1 in polysome fractions (Figure S2A-S2E). GLUT1 knockdown by siRNAs significantly inhibited the enhancement of glycolysis and the uptake of glucose by overexpression of BRIX1 in CRC cells (Figure 4H). Moreover, GLUT1 knockdown also inhibited increased the production of lactic acid by overexpression of BRIX1 in CRC cells (Figure 4I).

\section{BRIX1 plays a tumor-promoting role in CRC via regulating GLUT1 related glycolysis}

The effect of BRIX1 on CRC is still unclear. We knocked down BRIX1 in CRC cells (HCT-116 and SW480). In vitro experiments showed that BRIX1 knockdown significantly inhibited the proliferation of CRC cells (Figure 5A, 5B). Orthotopic transplantation experiments also showed BRIX1 significantly inhibited the growth of transplanted tumors (Figure $5 \mathrm{C}-5 \mathrm{E}, \mathrm{S} 3$ ). Furthermore, we overexpressed BRIX1 in CRC cells (HT29) and in vitro experiments showed that BRIX1 overexpression significantly promoted the proliferation of CRC cells (Figure 5F). Knockdown of GLUT1 with siRNA significantly inhibited the effect of BRIX1 overexpression on the growth of CRC cells (Figure 5F). Similarly, Block glycolysis by use of galactose can significantly reverse the effect of BRIX1 on the growth of CRC cells (Figure 5F).

\section{Up-regulated BRIX1 shows a close correlation with clinical features and associated with poor prognosis}

Based on the results of this study, we further analyzed the expression of BRIX1 in CRC, and the results showed that the mRNA and protein expression of BRIX1 were both significantly increased in CRC sample, 
compared with that in NC samples (Figure 6A-6D). The high expression of BRIX1 is closely related to the clinical characteristics of CRC patients, such as tumor size, T stage, and pathological stage (Figure 6E). In addition, data analysis also showed that the overall survival of CRC patients in the BRIX1 high expression group was significantly lower than that of the BRIX1 low expression patients (Figure 6F, 6G). These results all indicate that abnormally elevated BRIX1 expression plays an important role in CRC. Therefore, BRIX1 plays a tumor-promoting role in CRC via regulating glycolysis by selecting GLUT1 translation (Figure 6H).

\section{Discussion}

In human cells, ribosomes are relatively conserved organelles, composed of four types of RRNAs (5S, $5.8 \mathrm{~S}, 18 \mathrm{~S}$, and $28 \mathrm{~S}$ rRNA) and a large number of ribosome proteins (RPS). The synthesis process includes ribosome DNA (rDNA) transcription, precursor ribosomal RNA (pre-rRNA) formation, rRNA splicing and processing, assembly and transport of ribosomal subunits(4). In the nucleolus region, with the assistance of RNA polymerase Pol I and upstream binding factor (UBF), rDNA transcribed and synthesized to $47 \mathrm{~S}$ pre-rRNA (47S pre-rRNA). Three types of rRNAs (5.8S, $18 \mathrm{~S}$ and $28 \mathrm{~S}$ ) were formed through modification and splicing, while 5S rRNA was transcriptionally processed in the nucleoplasm by RNA polymerase Pol $\mathrm{III}(17,18)$. RPs are transcribed by RNA polymerase Pol II, and are further matured and transported to the nucleoli after translation, processing and modification. Then 18S rRNA and 33 kinds of RPs were assembled to form the 40S small subunit of the ribosome, while 5S rRNA, 5.8S rRNA and 28S rRNA were assembled with 47 kinds of RPs to form the $60 \mathrm{~S}$ large subunit of the ribosome. After further processing, assembly and transport, the large and small subunits were combined with mRNAs in the cytoplasm. Finally, mature ribosomal executive protein translation function is formed(4). Among them, rDNA transcription to form rRNA is crucial, which directly determines the rate of ribosome synthesis $(19,20)$. The transcriptional process of rRNA involves the activation of RNA polymerase Pol I/III, UBF recruitment, non-coding RNAs, regulation of nucleolins and ribosomal-related proteins, and splicing and processing of precursor RNAs, forming a complex molecular regulatory network, among which the interactions between related molecules are particularly important $(20,21)$. In this study, we first analyzed the enrichment of ribosomal function in $\mathrm{CRC}$, and the results showed that ribosomal pathways were significantly enriched in CRC, ranking among the top3. Further analysis found that BRIX1 gene may be the core gene of ribosomal pathway changes in CRC.

The yeast homologous of BRIX1 is Ribosome biogenesis protein Brx1 (Brx1), which has been reported to play an important role in the transcription of rRNA in yeast(22). In this study, due to bioinformatics analysis, we found BRIX1 was associated with ribosome function. BRIX1 knockdown significantly inhibited ribosome synthesis in CRC cells. Interestingly, in clinical related studies, we found that the glucose uptake value of PET-CT of CRC patients with high BRIX1 expression was significantly increased. Subsequent experiments found that BRIX1 knockdown significantly reduced the glycolytic ability of CRC cells. The glycolysis process of cells is catalyzed and regulated by a series of enzymes(23-25). Further research found that BRIX1 only affects the protein expression level of GLUT1, but not the protein 
expression of other enzymes, and BRIX1 did not affect the mRNA level of GLUT1. This indicates that the ribosome function regulated by BRIX1 may have some connection with the translation of GLUT1.

Ribosomes are not a static and homogeneous organelle, but a dynamic and heterogeneous complex. The protein translation function performed by it has obvious selectivity, called "ribosomal heterogeneity"(26). Ribosome heterogeneity is a broad concept, including changes in ribosomal composition and modification of rRNA and RPs(27). Studies have shown that there are quite differences in the types of RPs of ribosomes in eukaryotic cells, and these differences result in ribosomes being selective in translating mRNAs $(27,28)$. For example, ribosomes containing RPS25/eS25 or RPL10a/uL1 ribosomal proteins have different translation capabilities for hundreds of mRNAs(28). In this study, we found BRIX1 also promote the translation of more GLUT1 mRNA into protein.

There is a close relationship between the selective translation of ribosomes and cell metabolism $(29,30)$. On the one hand, the metabolic state of cells can directly affect the production of ribosomes and exert their physiological effects. The activation of the mTOR pathway affects the translation of downstream proteins by regulating the level of cellular glucose metabolism(31). On the other hand, the ribosome selectively regulates the process of mRNA translation into proteins and regulates the metabolic state of the cell. The selective translation of ribosomes can regulate the level of glucose metabolism in tumor cells $(32,33)$. In our research, BRIX1 promote the translation of more GLUT1 mRNA into protein. This explains the mechanism of selective action of ribosomes on glycolysis mechanism in some extent.

In this study, we found BRIX1 regulated ribosome function and promoted glycolysis by selected translation of GLUT1, which promoted CRC process.

\section{Conclusion}

In this study, we analyzed the changes of ribosome-related pathways in CRC and screened its key genes. Combined with clinical data, we found a correlation between BRIX1 and glucose uptake. Furthermore, BRIX1 regulated ribosome function and promoted glycolysis by selected translation of GLUT1, which promoted CRC process. This study explored the possibility of ribosome selectively regulating tumor cell metabolism, which provides theoretical support for targeted ribosome therapy in CRC.

\section{Abbreviations}

CRC: Colorectal cancer

BRIX1:Ribosome biogenesis protein BRX1 homolog

DMEM: Dulbecco's modified Eagle's medium

FBS: fetal bovine serum

shRNA: short-hairpin RNA 
BCA:bicinchoninic acid

H2O2:hydrogen peroxide

BSA:bovine serum albumin

DAB: 3,3-diaminobenzidine

ECAR: extracellular acidification rate

OCR: oxygen consumption rate

SD: standard deviation

PPI: protein protein interaction

RP: ribosome protein

rDNA: ribosome DNA

rRNA: ribosome RNA

UBF: upstream binding factor

\section{Declarations}

\section{Ethics approval and consent to participate}

The study was approved by the Research Ethics Committee of Renji Hospital and carried out in accordance with ethical standards as formulated in the Helsinki Declaration. Informed consents were provided by all patients.

\section{Consent for publication}

We all agree this manuscript publish in this journal.

\section{Availability of data and material}

I would like to declare on behalf of my co-authors that the work described was original research that has not been published previously, and not under consideration for publication elsewhere, in whole or in part. All data and material in this study were available.

\section{Competing interests}

The authors declare no potential conflicts of interest.

\section{Funding}


This study was supported by National Natural Science Foundation of China (82072671) and Research Fund for medical engineering cross project of Shanghai Jiao Tong University (ZH2018ZDA08).

\section{Authors' contributions}

$\mathrm{JCH}$ and $\mathrm{XCJ}$ carried out the molecular genetic studies, participated in the sequence alignment and in vitro and in vivo experiment. WSY participated in the sequence alignment. XQ and XHB participated in the design of the study and performed the statistical analysis. XQ and XHB conceived of the study, and participated in its design and coordination and helped to draft the manuscript. All authors read and approved the final manuscript.

\section{References}

1. Siegel RL, Miller KD, Fuchs HE, Jemal A. Cancer Statistics. 2021. CA: a cancer journal for clinicians. 2021;71(1):7-33.

2. Siegel RL, Miller KD, Goding Sauer A, Fedewa SA, Butterly LF, Anderson JC, et al. Colorectal cancer statistics, 2020. Cancer J Clin. 2020;70(3):145-64.

3. Weitz J, Koch M, Debus J, Hohler T, Galle PR, Buchler MW. Colorectal cancer. Lancet. 2005;365(9454):153-65.

4. Bassler J, Hurt E. Eukaryotic Ribosome Assembly. Annual review of biochemistry. 2019;88:281-306.

5. Kampen KR, Sulima SO, Vereecke S, De Keersmaecker K. Hallmarks of ribosomopathies. Nucleic acids research. 2020;48(3):1013-28.

6. Barbieri I, Kouzarides T. Role of RNA modifications in cancer. Nature reviews Cancer. 2020;20(6):303-22.

7. Sulima SO, Hofman IJF, De Keersmaecker K, Dinman JD. How Ribosomes Translate Cancer. Cancer discovery. 2017;7(10):1069-87.

8. Zhu B, Li Q, Liu R, Zheng M, Wen J, Zhao G. Genome-Wide Association Study of H/L Traits in Chicken. Animals: an open access journal from MDPI. 2019;9(5).

9. Faubert B, Solmonson A, DeBerardinis RJ. Metabolic reprogramming and cancer progression. Science. 2020;368(6487).

10. Yi M, Li J, Chen S, Cai J, Ban Y, Peng Q, et al. Emerging role of lipid metabolism alterations in Cancer stem cells. Journal of experimental clinical cancer research: CR. 2018;37(1):118.

11. Yoshida GJ. Metabolic reprogramming: the emerging concept and associated therapeutic strategies. Journal of experimental clinical cancer research: CR. 2015;34:111.

12. Datta M, Coussens LM, Nishikawa H, Hodi FS, Jain RK. Reprogramming the Tumor Microenvironment to Improve Immunotherapy: Emerging Strategies and Combination Therapies. American Society of Clinical Oncology educational book American Society of Clinical Oncology Annual Meeting. 2019;39:165 - 74. 
13. Vaupel $P$, Schmidberger $H$, Mayer $A$. The Warburg effect: essential part of metabolic reprogramming and central contributor to cancer progression. Int J Radiat Biol. 2019;95(7):912-9.

14. Weng ML, Chen WK, Chen XY, Lu H, Sun ZR, Yu Q, et al. Fasting inhibits aerobic glycolysis and proliferation in colorectal cancer via the Fdft1-mediated AKT/mTOR/HIF1alpha pathway suppression. Nature communications. 2020;11(1):1869.

15. Hu T, Liu H, Liang Z, Wang F, Zhou C, Zheng X, et al. Tumor-intrinsic CD47 signal regulates glycolysis and promotes colorectal cancer cell growth and metastasis. Theranostics. 2020;10(9):4056-72.

16. Xu C, Sun L, Jiang C, Zhou H, Gu L, Liu Y, et al. SPP1, analyzed by bioinformatics methods, promotes the metastasis in colorectal cancer by activating EMT pathway. Biomedicine pharmacotherapy $=$ Biomedecine pharmacotherapie. 2017;91:1167-77.

17. Pelletier $\mathrm{J}$, Thomas $\mathrm{G}$, Volarevic S. Ribosome biogenesis in cancer: new players and therapeutic avenues. Nature reviews Cancer. 2018;18(1):51-63.

18. Jung HM, Choi SJ, Kim JK. Expression profiles of SV40-immortalization-associated genes upregulated in various human cancers. Journal of cellular biochemistry. 2009;106(4):703-13.

19. Petelski AA, Slavov N. Analyzing Ribosome Remodeling in Health and Disease. Proteomics. 2020;20(17-18):e2000039.

20. Al-Shayeb B, Sachdeva R, Chen LX, Ward F, Munk P, Devoto A, et al. Clades of huge phages from across Earth's ecosystems. Nature. 2020;578(7795):425-31.

21. Kim DS, Challa S, Jones A, Kraus WL. PARPs and ADP-ribosylation in RNA biology: from RNA expression and processing to protein translation and proteostasis. Genes Dev. 2020;34(5-6):30220.

22. Kaser A, Bogengruber E, Hallegger M, Doppler E, Lepperdinger G, Jantsch M, et al. Brix from xenopus laevis and brx1p from yeast define a new family of proteins involved in the biogenesis of large ribosomal subunits. Biological chemistry. 2001;382(12):1637-47.

23. Liao M, Liao W, Xu N, Li B, Liu F, Zhang S, et al. LncRNA EPB41L4A-AS1 regulates glycolysis and glutaminolysis by mediating nucleolar translocation of HDAC2. EBioMedicine. 2019;41:200-13.

24. Deng F, Zhou R, Lin C, Yang S, Wang H, Li W, et al. Tumor-secreted dickkopf2 accelerates aerobic glycolysis and promotes angiogenesis in colorectal cancer. Theranostics. 2019;9(4):1001-14.

25. Schneditz G, Elias JE, Pagano E, Zaeem Cader M, Saveljeva S, Long K, et al. GPR35 promotes glycolysis, proliferation, and oncogenic signaling by engaging with the sodium potassium pump. Science signaling. 2019;12(562).

26. Genuth NR, Barna M. The Discovery of Ribosome Heterogeneity and Its Implications for Gene Regulation and Organismal Life. Molecular cell. 2018;71(3):364-74.

27. Segev N, Gerst JE. Specialized ribosomes and specific ribosomal protein paralogs control translation of mitochondrial proteins. J Cell Biol. 2018;217(1):117-26.

28. Shi Z, Fujii K, Kovary KM, Genuth NR, Rost HL, Teruel MN, et al. Heterogeneous Ribosomes Preferentially Translate Distinct Subpools of mRNAs Genome-wide. Molecular cell. 2017;67(1):71- 
83. e7.

29. Guo H. Specialized ribosomes and the control of translation. Biochemical Society transactions. 2018;46(4):855-69.

30. Wagner S, Herrmannova A, Hronova V, Gunisova S, Sen ND, Hannan RD, et al. Selective Translation Complex Profiling Reveals Staged Initiation and Co-translational Assembly of Initiation Factor Complexes. Molecular cell. 2020;79(4):546-60. e7.

31. Morita M, Gravel SP, Chenard V, Sikstrom K, Zheng L, Alain T, et al. mTORC1 controls mitochondrial activity and biogenesis through 4E-BP-dependent translational regulation. Cell Metabol. 2013;18(5):698-711.

32. Greenman IC, Gomez E, Moore CE, Herbert TP. The selective recruitment of mRNA to the ER and an increase in initiation are important for glucose-stimulated proinsulin synthesis in pancreatic betacells. Biochem J. 2005;391(Pt 2):291-300.

33. Manfrini N, Ricciardi S, Alfieri R, Ventura G, Calamita P, Favalli A, et al. Ribosome profiling unveils translational regulation of metabolic enzymes in primary CD4(+) Th1 cells. Dev Comp Immunol. 2020;109:103697.

\section{Figures}


A

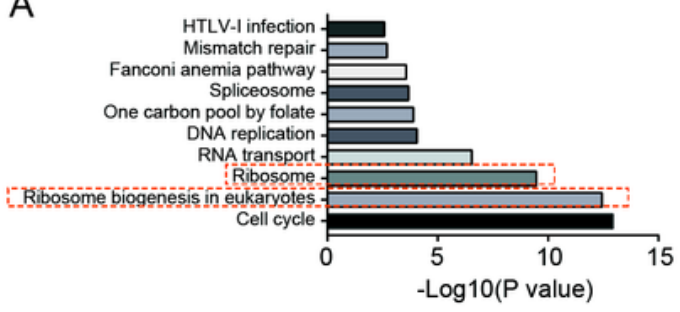

B

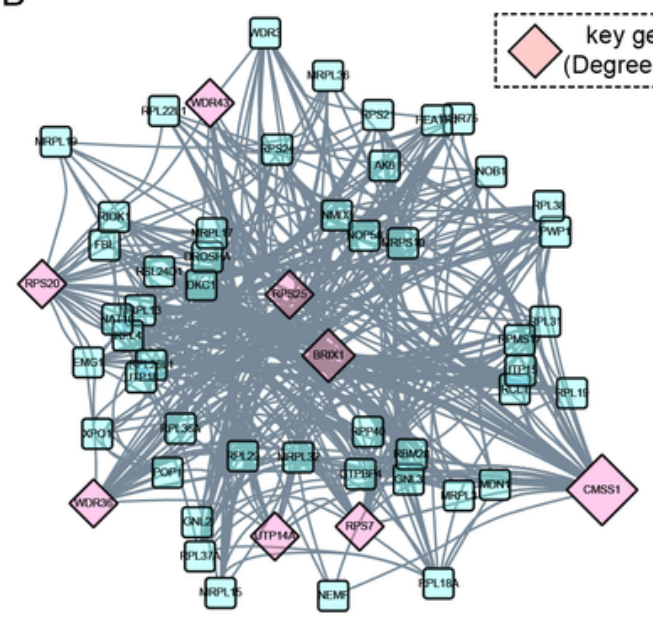

D
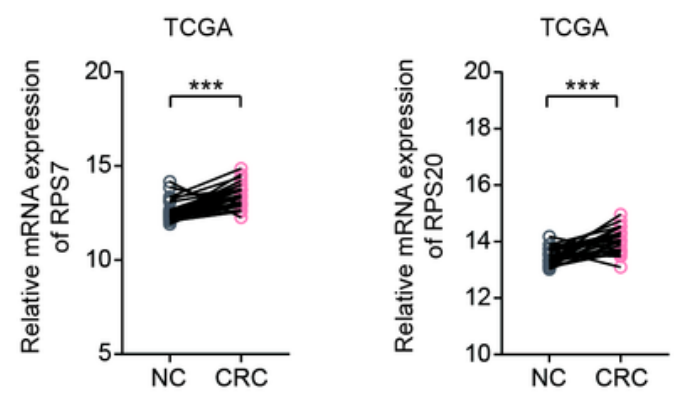

$\mathrm{E}$

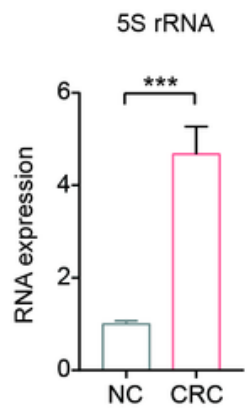

C
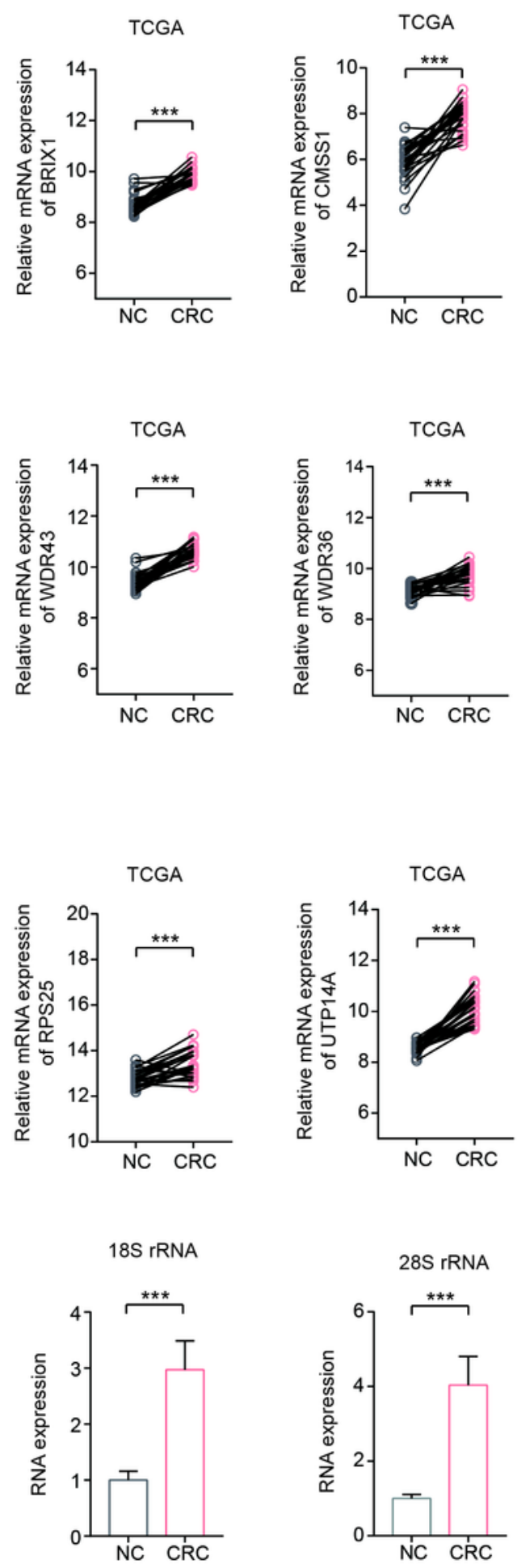

\section{Figure 1}

Ribosome-related pathways are primary enriched in CRC. A.KEGG pathway analysis of differentially expressed genes (FC>0.5 or FC<0.5) from GSE21510 and GSE18105 datasets in CRC. B. Key genes (degree>30) selection in ribosome-related pathways by cytoscope. C. mENA expression of BRIX1, CMSS1, WDR43 and WDR36 in CRC and paired adjacent tissues in TCGA dataset $(n=440)$. D. mENA expression of RPS7, RPS20, RPS25 and UTP14A in CRC and paired adjacent tissues in TCGA dataset (n=440). E. rRNAs 
(5S, 5.8S, 18S and 28S) levels in CRC and paired adjacent tissues ( $n=20)$. Measurement data were presented as the mean \pm SD. Student's t-test was used for statistical analysis. ${ }^{* \star *} \mathrm{P}<0.001$.

A

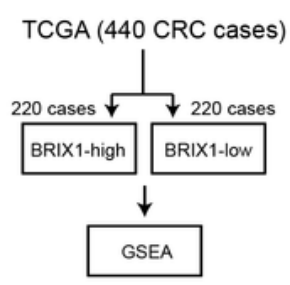

B

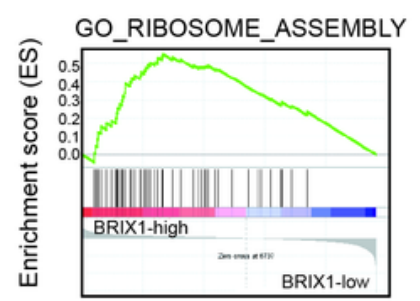

C

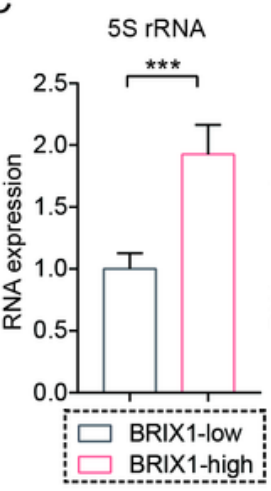

5.8S rRNA

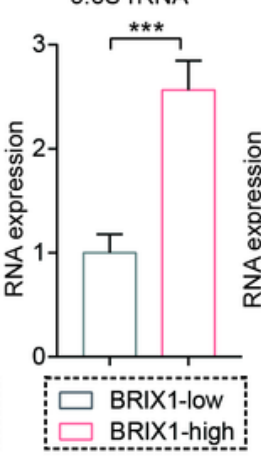

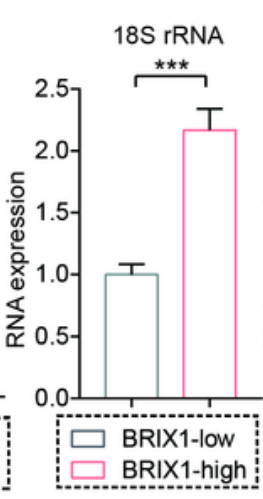

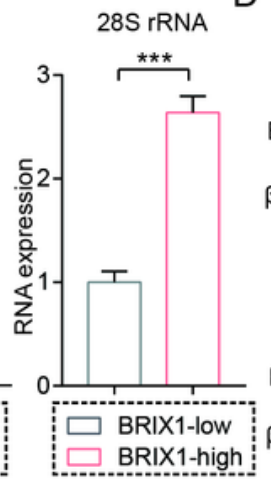

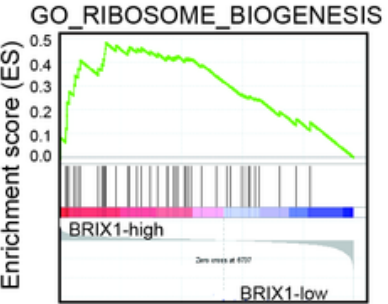

D

$\mathrm{E}$
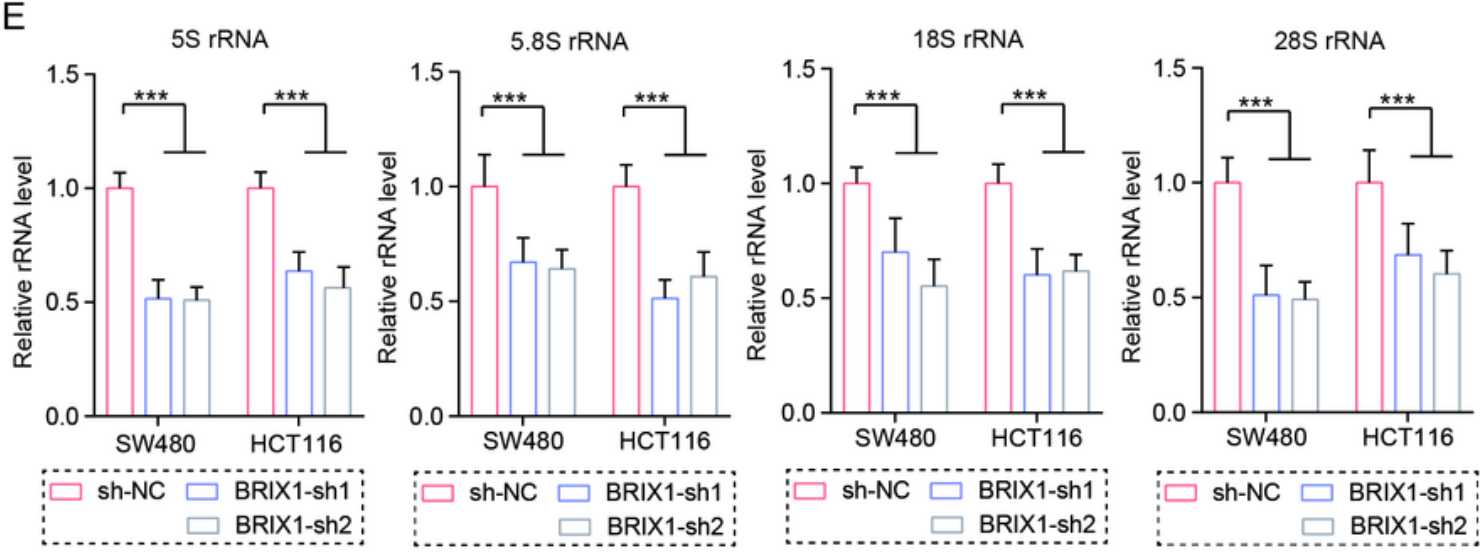

$\mathrm{F}$

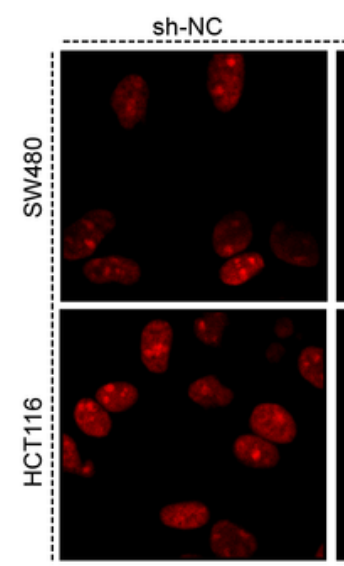

sh-BRIX1

BMH-21.
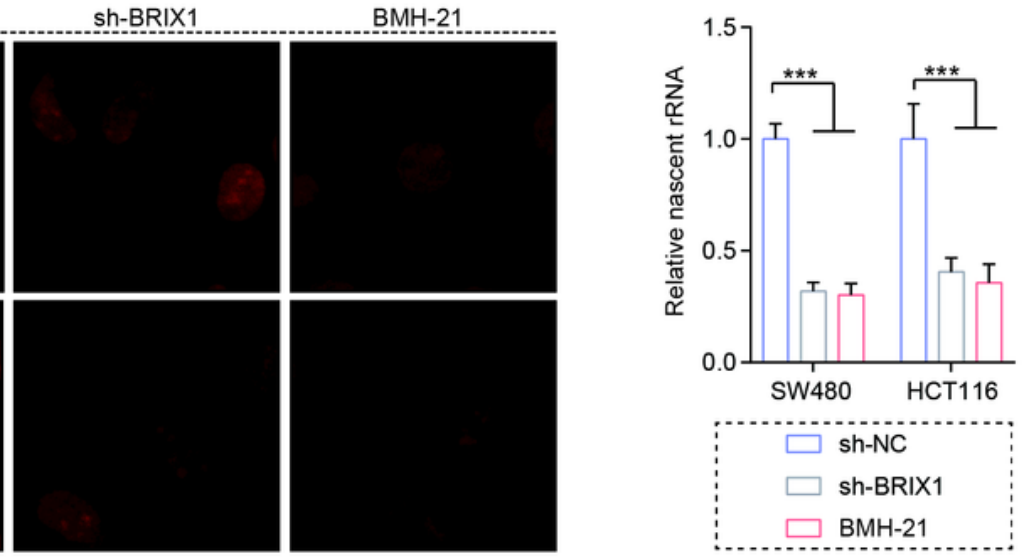

Figure 2

BRIX1 welly predicts ribosome activation in CRC. A and B.GSEA analysis of BRIX1 expression in CRC, as evaluated from TCGA dataset. A total of $440 \mathrm{CRC}$ samples were divided into two groups, including high BRIX1 expression group (220 samples) and low BRIX1 expression group (220 samples). C.rRNAs (5S, 
5.8S, 18S and 28S) levels in BRIX1-low and BRIX1-high CRC tissues ( $n=20)$. D. protein expression of BRIX1 in NCM460, RKO, HT29, SW620, SW480 and HCT-116 cell lines. BRIX1 knockdown in HCT-116 and SW480 cell lines by shRNAs. E. rRNAs (5S, 5.8S, 18S and 28S) levels in shNC and sh-BRIX1 HCT-116 and SW480 cells. F. Nascent RNA synthesis in shNC and sh-BRIX1 HCT-116 and SW480 cells, BMH-21 as a negative control. Measurement data were presented as the mean \pm SD. Student's t-test was used for statistical analysis. $* * * \mathrm{P}<0.001$.

A

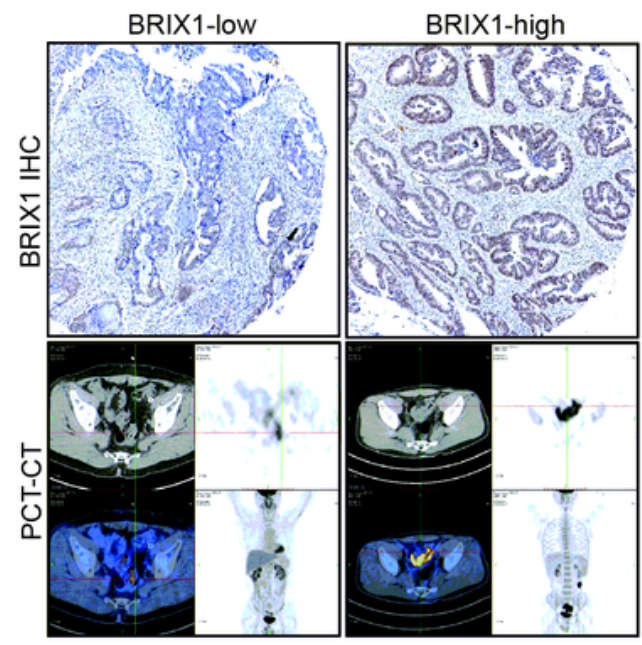

C

HCT-116

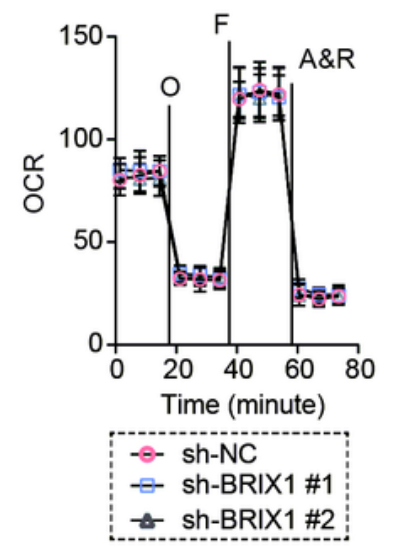

D

HCT-116

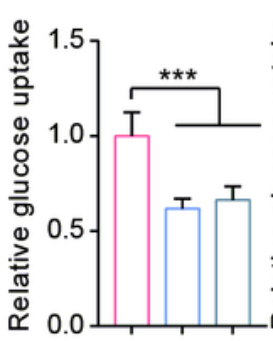

$\square$ sh-NC
$\square$ sh-BRIX1 \#1
$\square$ sh-BRIX1 \#2

$\square$ sh-BRIX1 \#2
SW480

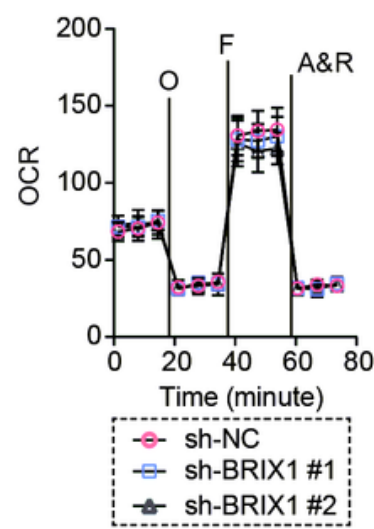

E

SW480

HCT-116

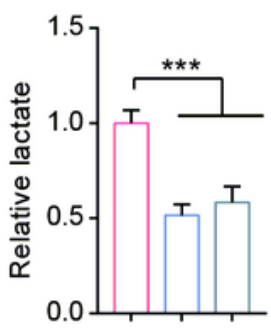

$\square$ sh-NC

$\square$ sh-BRIX1 \#1 sh-BRIX1 \#2
B
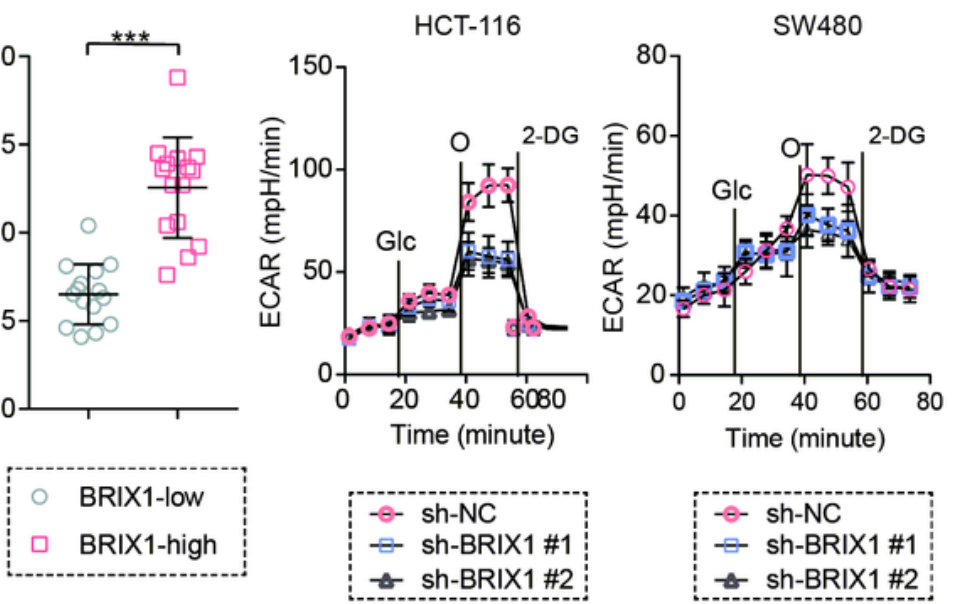

$-\Theta-$ sh-NC

- sh-BRIX1\#1

- sh-BRIX1\#2

F

HT-29

HT-29
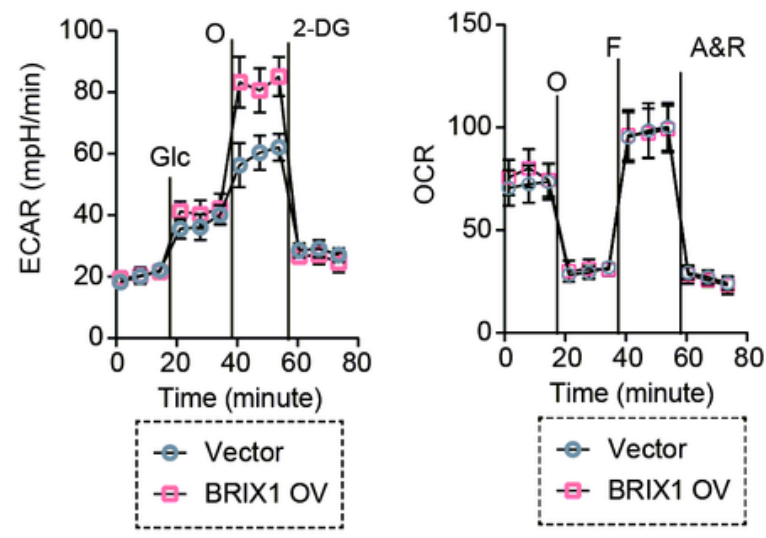

G

SW480

HT-29

HT-29
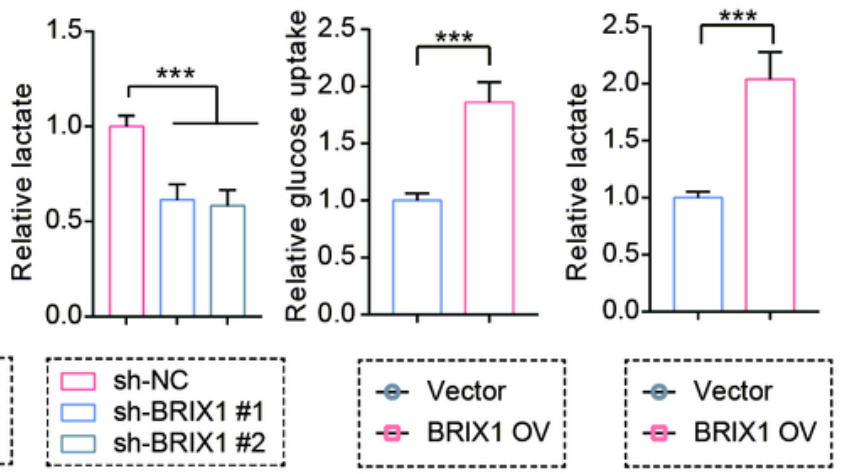

Figure 3 
BRIX1 determines a high glycolytic state in CRC cells. A. PET-CT images in low expression of BRIX1 and high expression of BRIX1 CRC patients $(n=15)$. B. Extracellular acidification rate (ECAR) of HCT-116 or SW480 cells in the sh-NC and sh-BRIX1 group was detected via a Seahorse Bioscience XFp analyzer. Glc: glucose, 0: oligomycin, 2-DG: 2-deoxy-d-glucose. C. 02 consumption rate (OCR) of HCT-116 or SW480 cells in the sh-NC and sh-BRIX1 group was detected via a Seahorse Bioscience XFp analyzer. O:

Oligomycin, F: FCCP, A\&R: antimycin A/rotenone. D. ECAR and OCR of HT29 cells in the Vector and BRIX1 OV group was detected via a Seahorse Bioscience XFp analyzer. E. Glucose uptake of HCT-116 or SW480 cells in the sh-NC and sh-BRIX1 group. F. Lactic acid formation of HCT-116 or SW480 cells in the sh-NC and sh-BRIX1 group. G. Glucose uptake and lactic acid formation of HT29 cells in the Vector and BRIX1 OV group. Measurement data were presented as the mean \pm SD. Student's t-test was used for statistical analysis. ${ }^{\star \star \star} \mathrm{P}<0.001$. 
A

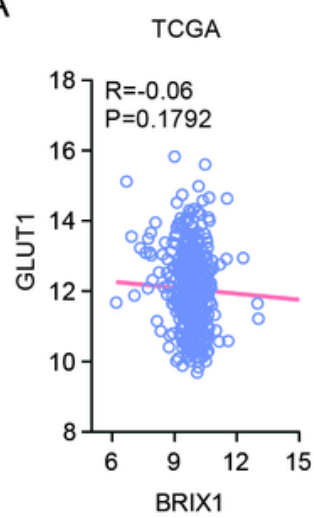

D

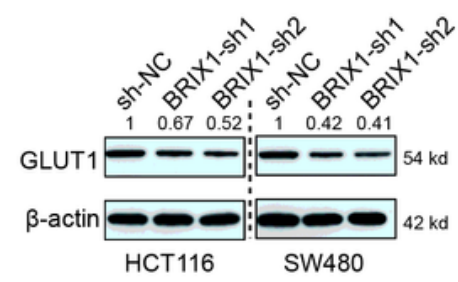

G
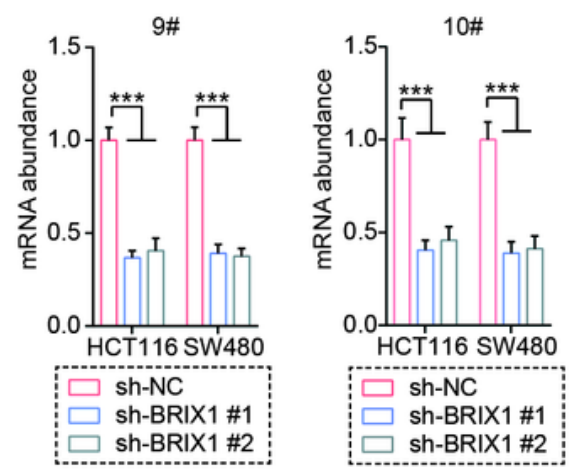

C

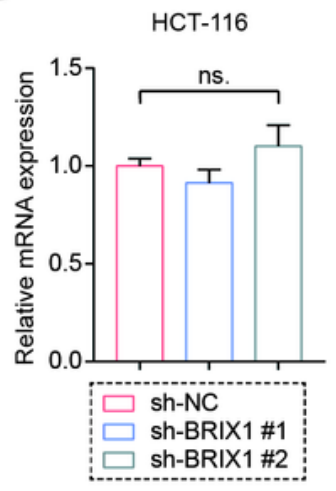

E
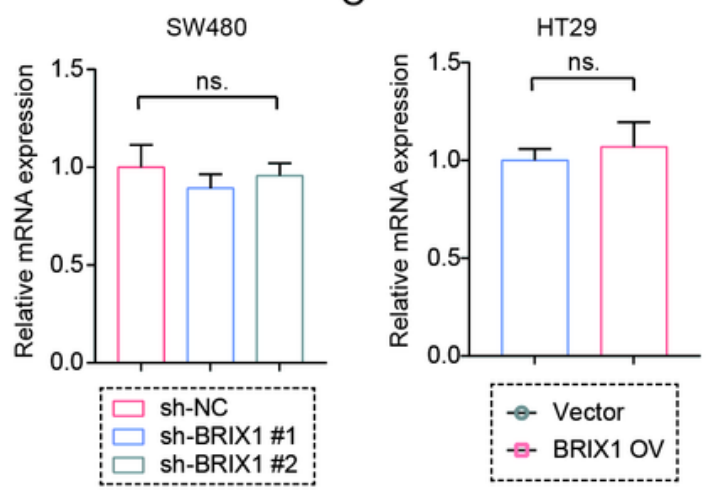

F
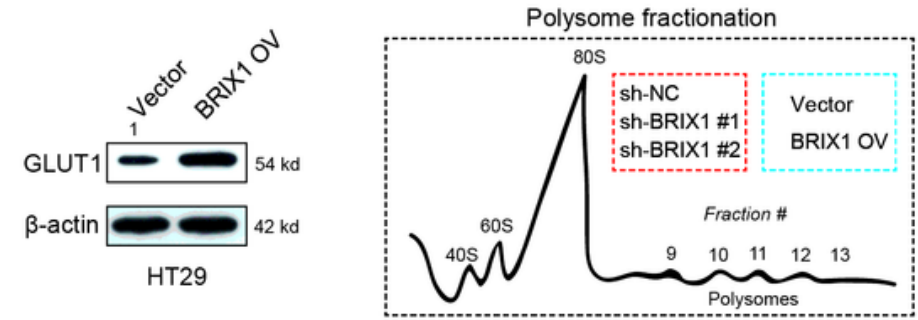

$\mathrm{H}$

HT-29

HT-29
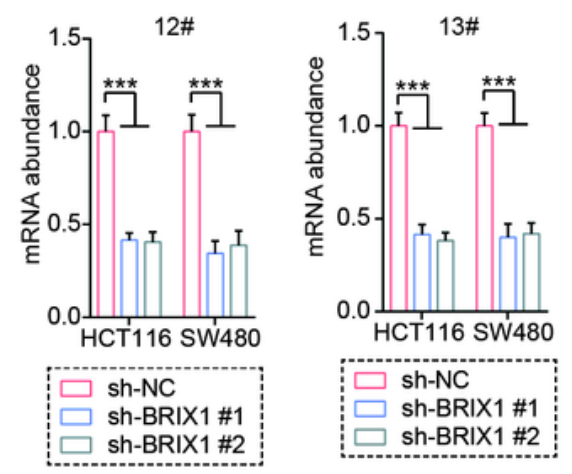

I
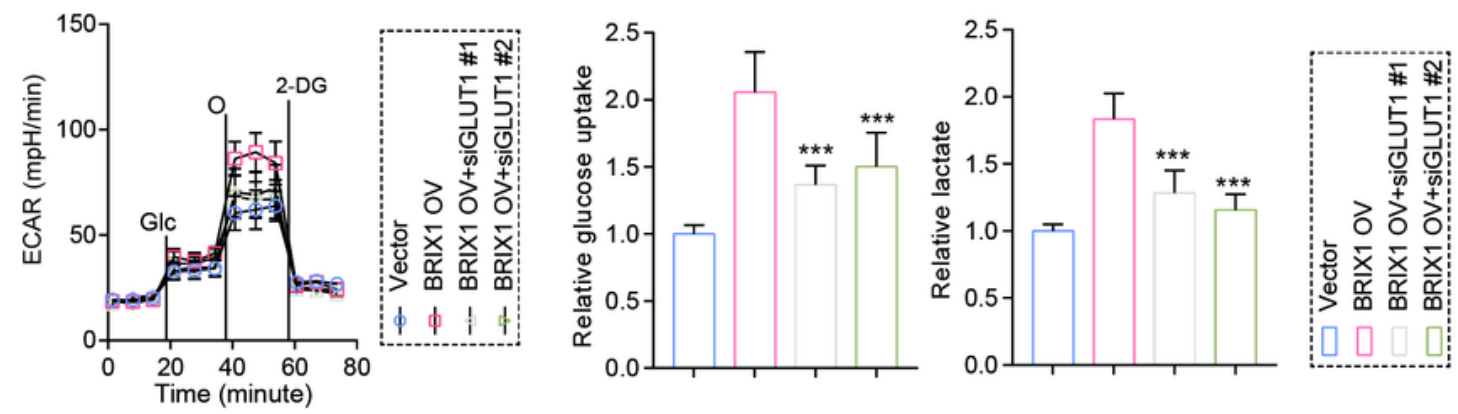

\section{Figure 4}

BRIX1 regulates glycolysis in CRC cells via selected translation for GLUT1. A.Correlation between BRIX1 and GLUT1 mRNA expression in TCGA datasets. B. mRNA expression of BRIX1 of HCT-116 or SW480 cells in the sh-NC and sh-BRIX1 group. C. mRNA expression of BRIX1 of HT29 cells in the Vector and BRIX1 OV group. D. Protein expression of HCT-116 or SW480 cells in the sh-NC and sh-BRIX1 group. E. Protein expression of BRIX1 of HT29 cells in the Vector and BRIX1 OV group. F. Polysome fractionation 
analysis in in the sh-NC and sh-BRIX1 group in HCT-116 and SW480 cellsCRC cells. G. mRNA abundance of GLUT1 in different polysome fractionations in the sh-NC and sh-BRIX1 group. H. ECAR of HT29 cells in the Vector and BRIX1 OV group with the treatment of siGLUT1 was detected via a Seahorse Bioscience XFp analyzer. Glc: glucose, O: oligomycin, 2-DG: 2-deoxy-d-glucose. I. Glucose uptake and lactic acid formation of HT29 cells in the Vector and BRIX1 OV group with the treatment of siGLUT1. Measurement data were presented as the mean \pm SD. Student's t-test was used for statistical analysis. ns. represents no statistical difference, ${ }^{* \star *} \mathrm{P}<0.001$.

A

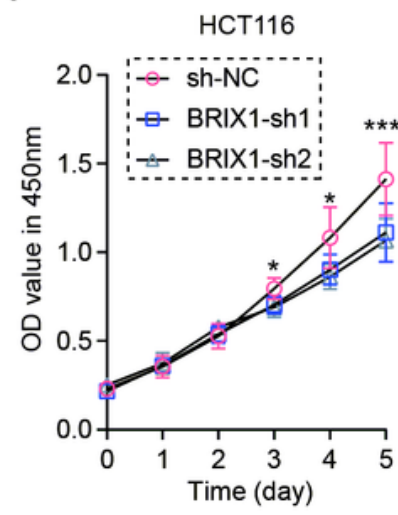

B

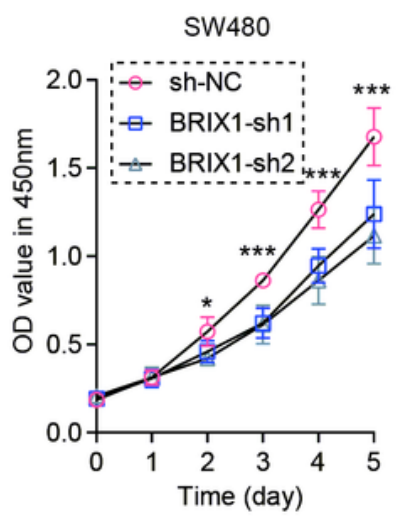

C

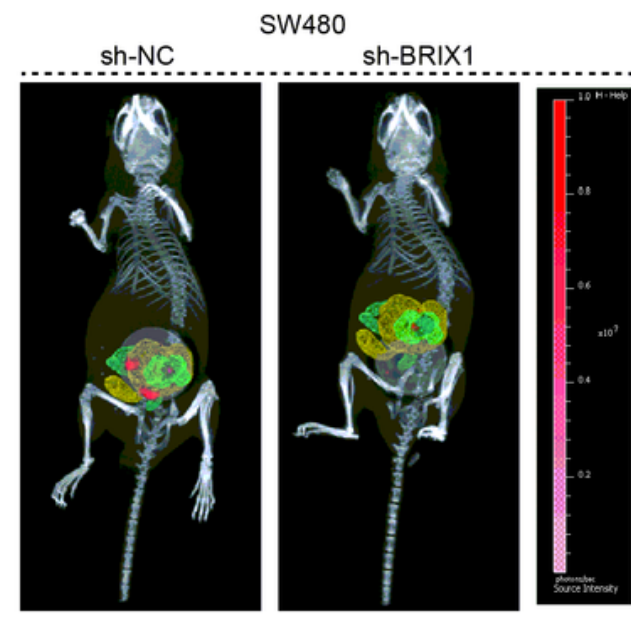

EdU assay (HCT116)
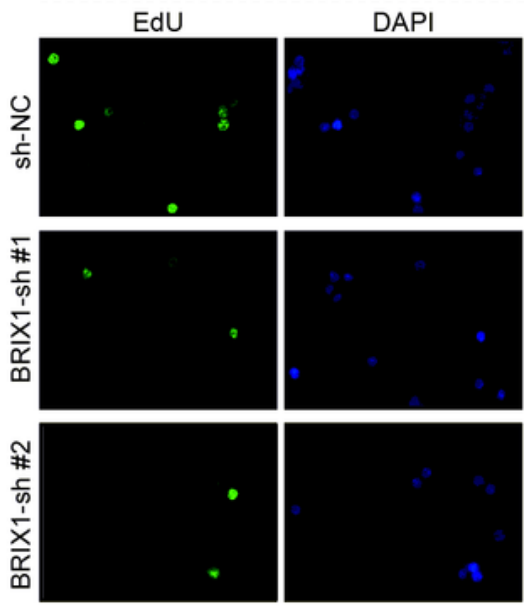

EdU assay (SW480)
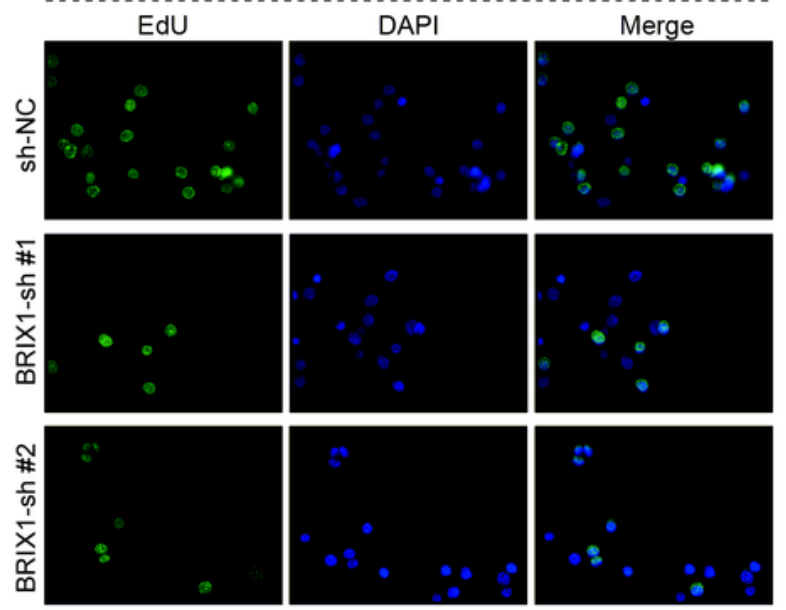

Merge

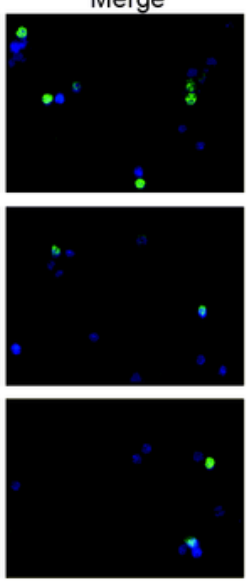

$\mathrm{E}$
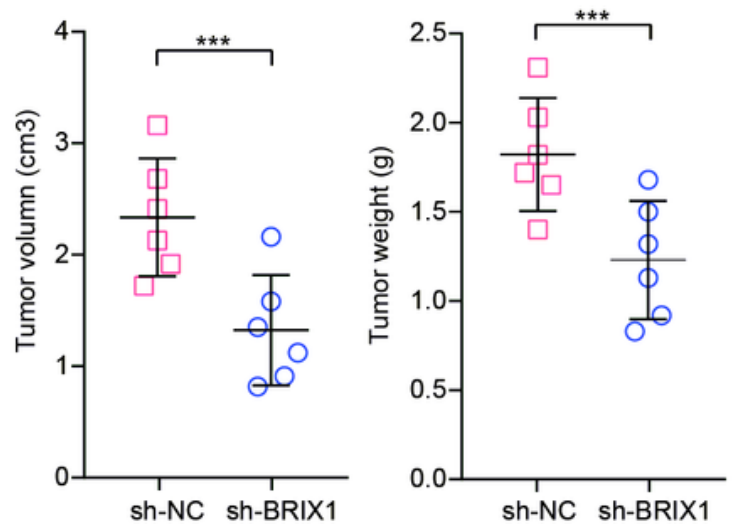

$\mathrm{F}$

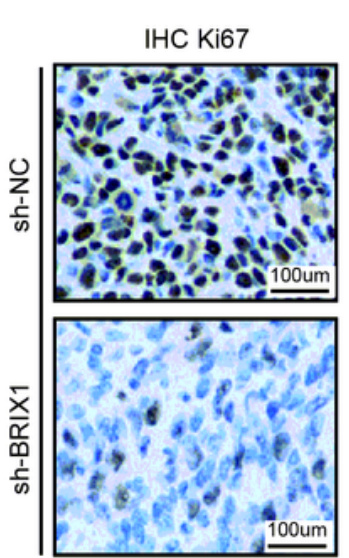

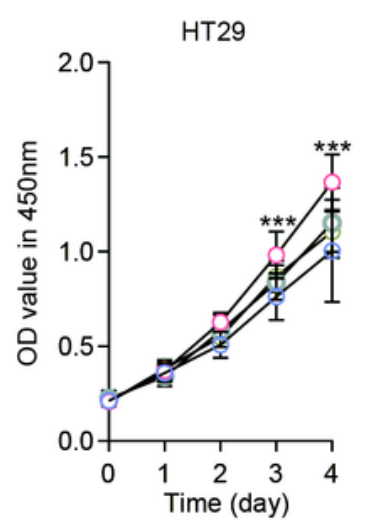

- - Vector

$\sim-$ BRIX1 OV

-o- BRIX1 OV+siGLUT1

- - BRIX1 OV+galactose 


\section{Figure 5}

BRIX1 plays a tumor-promoting role in CRC via regulating GLUT1 related glycolysis. A. The viability of HCT-116 and SW480 cells transfected with sh-BRIX1 or sh-control, as analyzed with CCK-8 assay. B.The viability of HCT-116 and SW480 cells transfected with sh-BRIX1 or sh-control, as analyzed with EdU assay. C. CT imaging of SW480luc cells transfected with sh-BRIX1 or sh-NC by orthotopic tumor assay $(n=6)$. D. Comparison of orthotopic tumor volume and weight between sh-NC group and sh-BRIX1 group. E. Expression of Ki-67 in the orthotopic tumor tissues (sh-NC group [ $n=6]$ and sh-BRIX1 group [n=6]). F. The viability of HT29 cellsin the Vector and BRIX1 OV group with the treatment of siGLUT1 and galactose, as analyzed with CCK-8 assay. Measurement data were presented as the mean \pm SD. Student's t-test was used for statistical analysis. ${ }^{*} P<0.05, * \star * P<0.001$. 
A

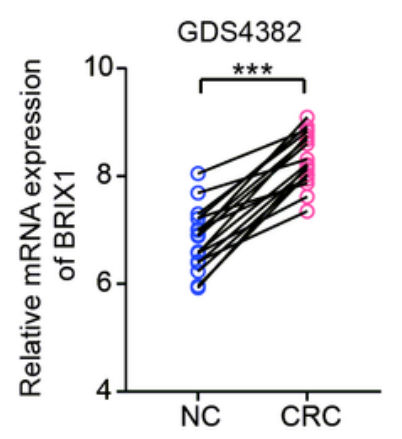

D

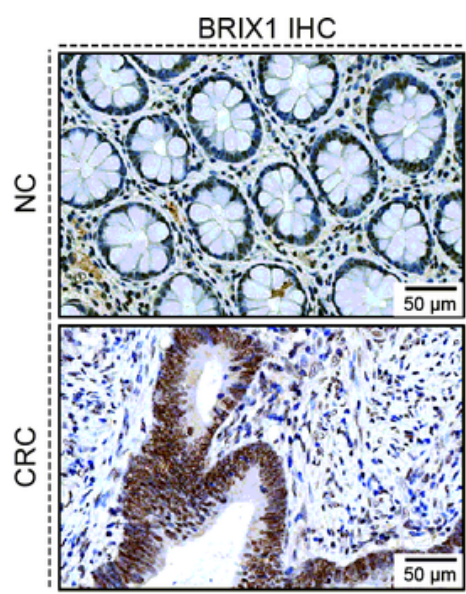

F

TCGA

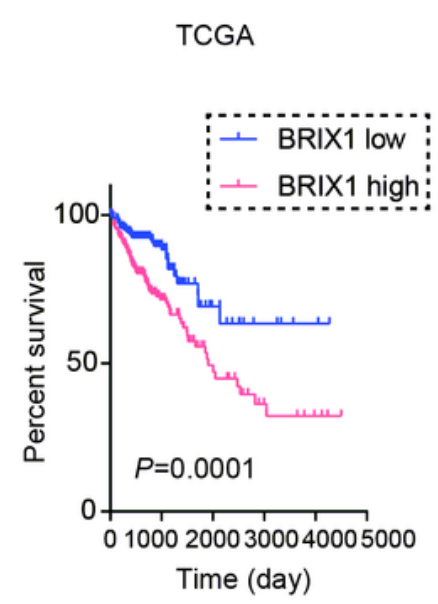

B

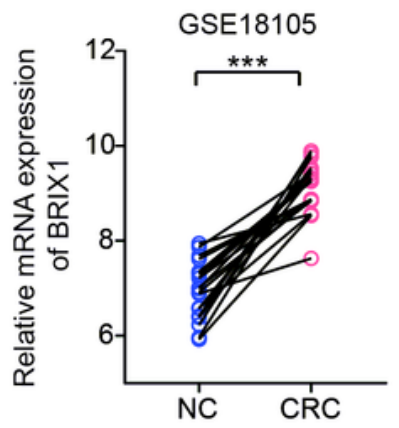

E
C

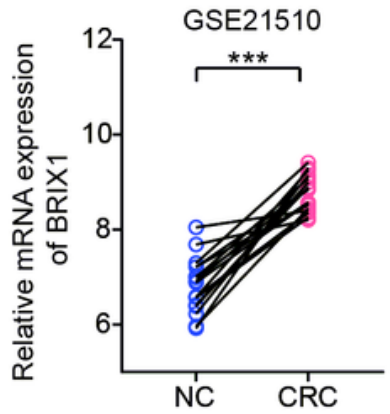

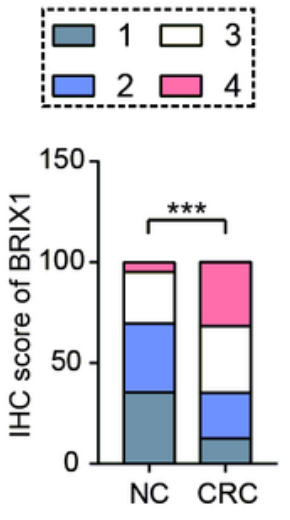

G

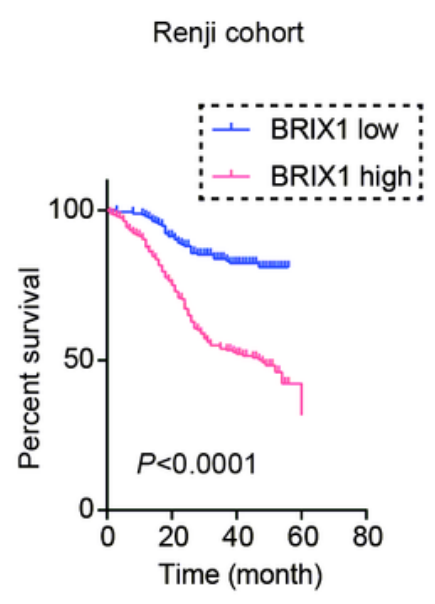

TCGA

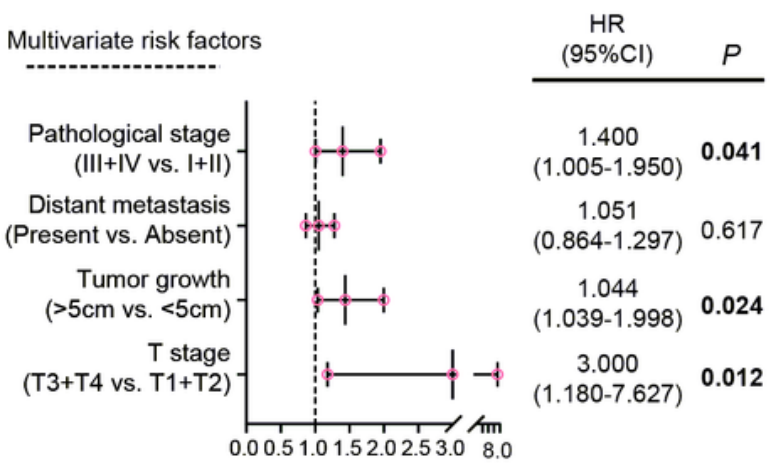

$\mathrm{H}$

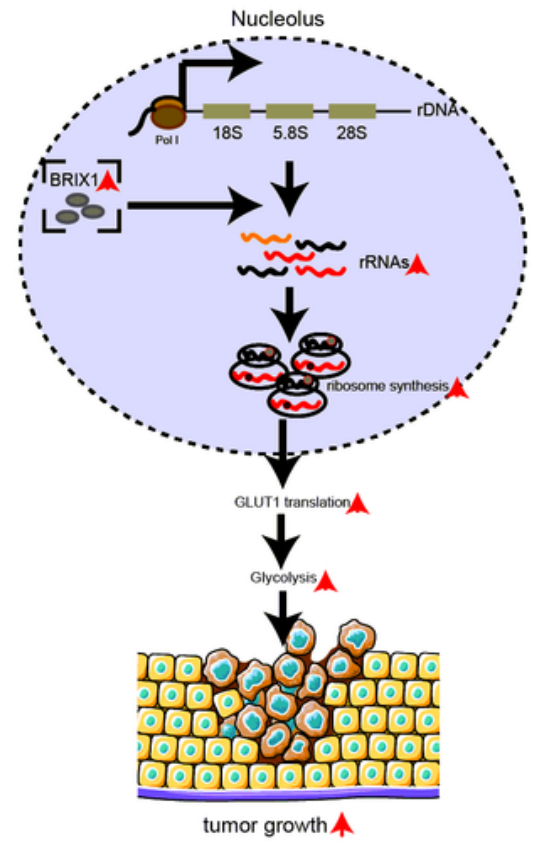

\section{Figure 6}

Up-regulated BRIX1 shows a close correlation with clinical features and associated with poor prognosis. A. mENA expression of BRIX in CRC and paired adjacent tissues in GDS4382 dataset $(n=17)$. B. mENA expression of BRIX in CRC and paired adjacent tissues in GSE18105 dataset $(n=20)$. C. mENA expression of BRIX in CRC and paired adjacent tissues in GSE20510 dataset ( $n=17)$. D. BRIX1 protein expression in CRC tissues from tissue microarray $(n=392)$. E. Clinical features analysis in low expression of BRIX1 and 
high expression of BRIX1 CRC tissues. F. Overall survival analysis based on the mRNA expression of BRIX1 from TCGA dataset. G. Overall survival analysis of the protein expression of BRIX1 based on the prognostic information of patients with CRC from tissue microarray data. H. BRIX1 plays a tumorpromoting role in CRC via regulating glycolysis by selecting GLUT1 translation. Measurement data were presented as the mean \pm SD. Student's t-test was used for statistical analysis. ${ }^{* *} \mathrm{P}<0.001$.

\section{Supplementary Files}

This is a list of supplementary files associated with this preprint. Click to download.

- supplementarymaterial.docx

- FigureS1.tif

- FigureS2.tif

- Figures3.tif

- tableS1.docx 